\title{
Topographic Characterization of Disposable Carbon Pencil Modified Electrodes
}

\author{
J. Berbejillo, ${ }^{a}$ J. Laiz, ${ }^{\text {a }}$ M.F. Cerdá, ${ }^{\text {M }}$ M.E. Martins, ${ }^{c}$ E. Méndez, ${ }^{b}$, \\ ${ }^{a}$ Unidad de Bioquímica Analítica. Centro de Investigaciones Nucleares, Facultad de Ciencias. \\ Universidad de la República, Montevideo, Uruguay \\ ${ }^{b}$ Laboratorio de Biomateriales. Instituto de Química Biológica. Facultad de Ciencias, \\ Universidad de la República. Iguá 4225 casi Mataojo. 11400 Montevideo, Uruguay \\ ${ }^{c}$ Instituto de Investigaciones Fisicoquímicas Teóricas y Aplicadas, Universidad Nacional de La \\ Plata, Provincia de Buenos Aires, Argentina
}

Received 23 March 2004; accepted in revised form 31 May 2004

\begin{abstract}
Carbon pencil electrodes are proposed as supporting material for the fabrication of disposable devices through surface modification by bismuth plating and polyaniline formation. The surface characteristics were assessed by fractal analysis under semiinfinite linear diffusion employing a soluble molecular probe, and scanning electron microscopic analysis. The results indicate that the surface topography for bismuthmodified electrodes corresponds to a 2-dimensional smooth surface, while for polyaniline-modified electrodes, a surface fractal dimension of 2.76 was obtained. These topographic characteristics indicate that bismuth-modified carbon electrodes are suitable for anodic stripping analysis, and polyaniline ones, due to the surface irregularities, may result a good supporting material for biomolecules attachment.
\end{abstract}

Keywords: carbon pencil electrodes, fractals, Randles-Sevcik, topography.

\section{Introduction}

The development of disposable sensors is one of the demanding areas in electroanalysis. These devices should meet several requirements, such as low cost, ease of fabrication and good performance. Although most of the papers on this subject have put attention mainly on the electronic properties of the sensors, their topography has not been considered in depth.

* Corresponding author. E-mail address: emendez@fcien.edu.uy 
Two different situations may be considered regarding the topography of electrochemical sensors. If such device is going to be used in stripping analysis of heavy metals, a smooth 2D surface is needed [1] in order to ensure the even deposition of the target metals. On the other hand, the development of modified surfaces for the attachment of proteins for the fabrication of biosensors needs a rough surface that enables the highest amount of adsorbed protein to be reached [2].

A common practice to describe the surface topography is the determination of its fractal dimension. The fractal dimension of an object is a number that coincides with the Hausdorff dimension, and this number allows the description of the surface topography within the non-euclidean geometry [3]. An object presenting a fractal dimension of 2 or 3 can be considered an euclidean object with perfect 2D and 3D dimension; non-integer values of the fractal dimension correspond to a fractal object.

In this work, a carbon pencil electrode (CPE) was chosen as the disposable platform for the design of two different sensors: a bismuth-plated electrode for stripping analysis and a polyaniline-coated sensor for biosensor design. In both cases, the fractal dimensions of the resulting surfaces were determined by electrochemical methods. It will be shown that the topographic characteristics of the resulting sensors are adequate for the planned analytical purposes.

\section{Materials and methods}

\section{Apparatus and electrodes}

Stripping voltammetry and current transients were performed employing a potentiostat (LYP-M7, Argentina) and a wave-function generator (LYP-G, Argentina), and were registered using a Linseis LY1600 Y-X-t recorder. A carbon pencil lead HB (Pilot), $1.0 \mathrm{~mm}$ diameter, was used as purchased, and only the bottom of the lead, previously polished over a filter paper, was exposed to the solution leaving an exposed geometric area of approximately $0.03 \mathrm{~cm}^{2}$. A graphite rod and an $\mathrm{Ag}|\mathrm{AgCl}| 3 \mathrm{M} \mathrm{KCl}(\mathrm{aq})$ were utilized as counter and 
reference electrode, respectively. Potentials in the text are referred to $\mathrm{Ag} / \mathrm{AgCl}$ reference electrode and all the experiments were performed at $298 \mathrm{~K}$.

\section{Reagents and solutions}

$\mathrm{Bi}\left(\mathrm{NO}_{3}\right)_{3}$ was used to prepare a $1000 \mathrm{mg} \mathrm{L}^{-1} \mathrm{Bi}$ standard solution, which was further diluted in $0.1 \mathrm{M}$ sodium acetate buffer solution $(\mathrm{pH}=4.6)$. Aniline was purified by column chromatography in $\mathrm{Al}_{2} \mathrm{O}_{3}$, and dissolved in $1.2 \mathrm{M}$ aqueous $\mathrm{HCl}$ to yield a $0.2 \mathrm{M}$ solution. Probe solutions containing $0.002 \mathrm{M} \mathrm{K}_{4}\left[\mathrm{Fe}(\mathrm{CN})_{6}\right]$ or methyl viologen dication $\left(\mathrm{MV}^{2+}\right)$ in $0.2 \mathrm{M} \mathrm{NaNO}$ were prepared. All solutions were prepared with MilliQ* water and analytical grade reagents.

\section{Preparation of Bi-plated electrodes}

Bismuth-plated carbon pencil electrodes (CPE-Bi) were obtained by deposition of metallic $\mathrm{Bi}$ from a $500 \mathrm{ppm}$ solution in acetate buffer $(\mathrm{pH}=4.6)$ in the presence of dissolved oxygen. The metal electrodeposition was accomplished at $-1.20 \mathrm{~V}$ under constant stirring with a preconcentration step of 2 min [4]. Therewith, the stirring was suspended for $30 \mathrm{~s}$ (rest period) and a voltammogram resulting from the application of a positive-going potential sweep up to $E_{a s}=$ $+0.3 \mathrm{~V}$ at $v=0.3 \mathrm{~V} \mathrm{~s}^{-1}$ was recorded.

\section{Preparation of PANI-coated electrodes}

Polyaniline-covered carbon pencil electrode (CPE-PANI) was prepared by in situ electropolymerization of the aniline monomer by potentiodynamic cycling in aqueous $\mathrm{HCl}$ media. The first potentiodynamic cycle was performed between $-0.15 \mathrm{~V}$ and $1.20 \mathrm{~V}$, in order to produce the radical anion that produces the polyaniline polymer by further cycling between $-0.15 \mathrm{~V}$ and $0.70 \mathrm{~V}$. Upon continuing cycling at $0.1 \mathrm{~V} \mathrm{~s}^{-1}$, the polymerization process proceeds, and the concomitant increment in the cathodic and anodic charges can be followed through the $i-E$ profile. 


\section{Fractal characterization of the electrodes}

In order to assess the fractal characteristic of the resulting modified electrodes, the method of Stromme et al. [5] was employed. Briefly, the voltamperometric behavior of a soluble molecular probe is studied under the conditions of semiinfinite linear diffusion in quiet solutions at different scan rates. The relationship between peak current vs. potential sweep rate is studied through a log-log plot, and the slope is related to the fractal dimension $\left(D_{\mathrm{f}}\right)$ through:

$$
\text { slope }=\frac{D_{f}-1}{2}
$$

For PANI-covered electrodes, $\mathrm{Fe}(\mathrm{CN})_{6}{ }^{3-}$ was employed as the electroactive molecular probe, while for Bi-coated electrodes, methyl viologen $\left(\mathrm{MV}^{2+}\right)$ was chosen because its redox potential is lower than that corresponding to $\mathrm{Bi}$ anodic stripping [6]. For the naked CPE, both molecular probes were employed. Once prepared, the modified electrodes were thoroughly washed with MilliQ* water and placed in the molecular probe-containing solution. Voltammograms were performed at different potential sweep rates $(v)$ in the $0.005 \leq v \leq 0.10 \mathrm{~V} \mathrm{~s}^{-1}$ range, after a potential step in the double layer region until a zero current was achieved, and the first scan was taken in every case. Cathodic current peaks $\left(i_{\text {cat }}\right)$ data were obtained after correction for the double layer current contribution.

\section{Results}

The topographical features of the naked CPE electrode were assessed by scanning electron microscopy (Fig. 1). Large areas with a smooth topography partially superposed between them can be seen in the micrograph. White little areas, preferentially present at the border of the plates correspond to the nonconducting organic binder used in the fabrication of the carbon pencil.

The relationship between the cathodic current peak and the square root of the sweep rate for naked CPE and CPE-Bi yields straight lines with zero intercept, in accordance to Randles-Sevcik equation (Fig. 2a). According to this equation, a perfectly smooth 2D surface should arise for both electrodes. Taking a value of $8.6 \times 10^{-6} \mathrm{~cm}^{2} \mathrm{~s}^{-1}$ for the diffusion coefficient of $\mathrm{MV}^{2+}$ [7] the calculated real 
areas for CPE and CPE-Bi were $0.039 \mathrm{~cm}^{2}$ and $0.040 \mathrm{~cm}^{2}$, respectively. The slight departure of the calculated real area of CPE from the geometric area can be attributed to the presence of the organic binder.

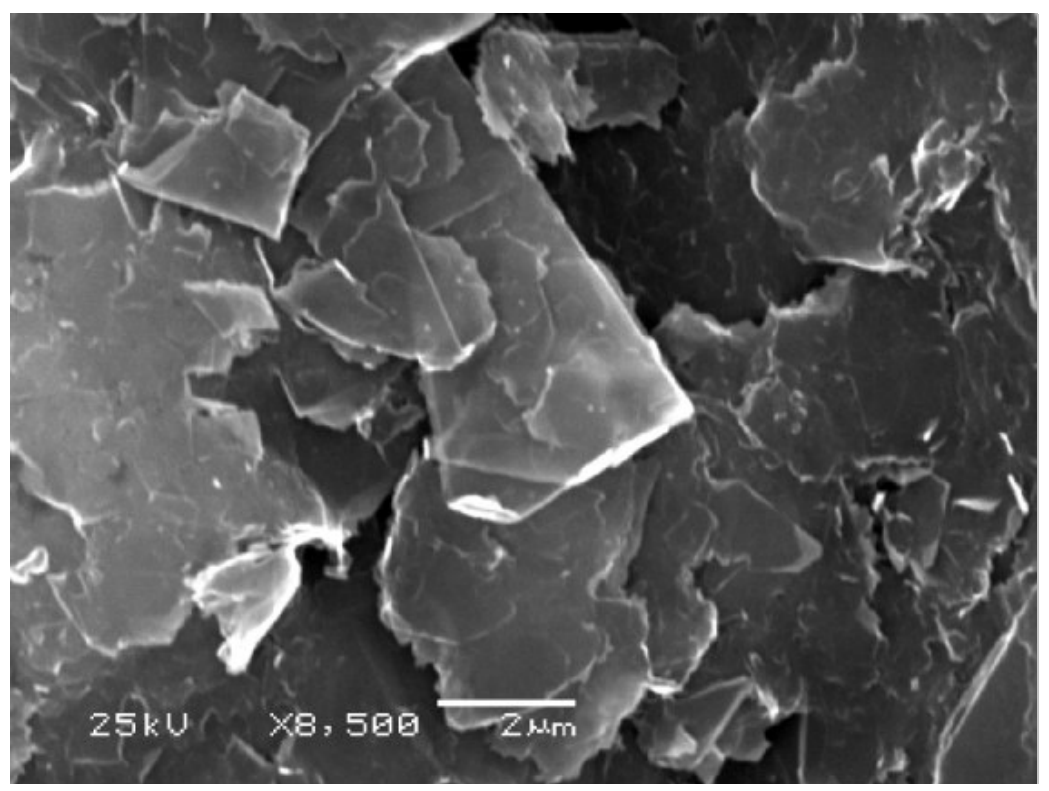

Figure 1. Scanning electron micrograph of a carbon pencil surface. Acceleration voltage: $25 \mathrm{kV}$, magnification: $x 8500$.

The log-log relationship between the cathodic current peak and the sweep rate confirms this situation. In both cases, good linear relationships were obtained $(r>0.999)$, with slopes of $0.494 \pm 0.005$ for CPE and $0.49 \pm 0.01$ for CPE-Bi (Fig. 2b). These figures correspond to fractal dimensions of $1.99 \pm 0.01$ and $1.98 \pm 0.02$, respectively. In order to gain more information on the growth mode of the Bi deposit, a chronoamperometric curve at $-1.2 \mathrm{~V}$ was performed. Three current contributions reproduce the experimental curve (Fig. 3), namely an exponential current decay corresponding to the double layer discharge, a 2D growth mode and a 3D under charge transfer conditions growth mode.

The topographic characteristics of CPE-PANI were assessed by scanning electron microscopy (Fig. 4). A network of tubular fibers can be seen, allowing the existence of several holes of diameters between $300 \mathrm{~nm}$ and 1.5 $\mu \mathrm{m}$. 


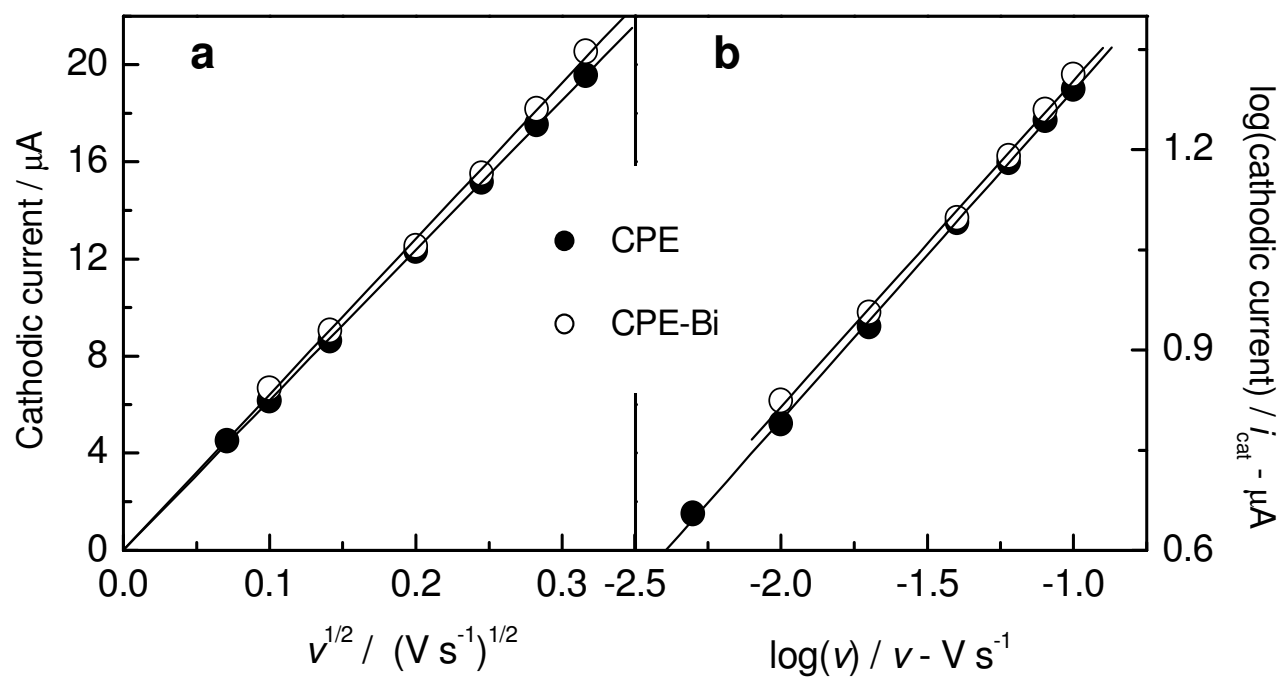

Figure 2. (a) Cathodic current vs. (sweep rate) ${ }^{1 / 2}$ plot for carbon pencil electrode (CPE) and bismuth-coated carbon pencil electrode (CPE-Bi). The linear relationship obtained is indicative of the accomplishment of the Randles-Sevcik equation. (b) log-log plot for data displayed in (a), for the fractal analysis according to Stromme et al. [5].

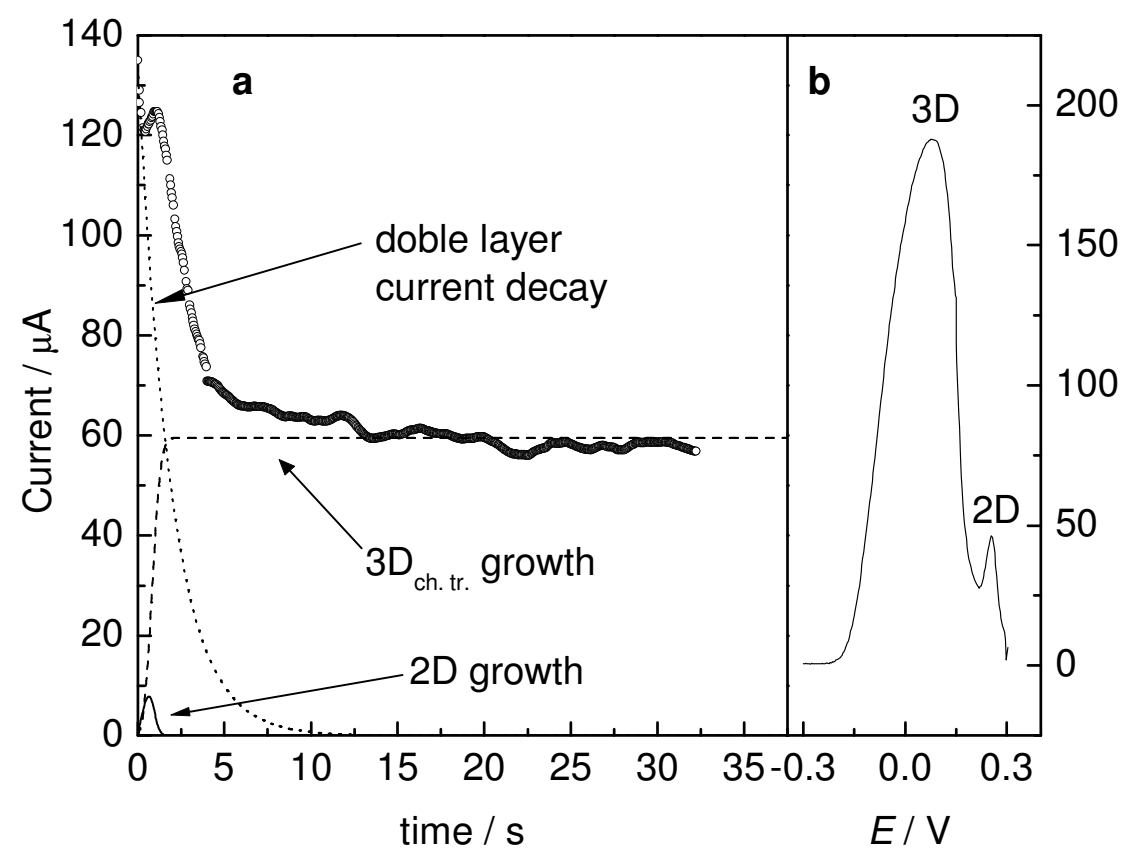

Figure 3. (a) Current transient obtained at $-1.2 \mathrm{~V}$ during the electrodeposition of bismuth onto a carbon pencil electrode. Experimental points $(\bigcirc)$, and current contributions obtained by graphical deconvolution (double layer current decay, ….....; 2D - ; and 3D ----- growths). (b) Anodic current profile for bismuth stripping from the carbon pencil electrode surface. 


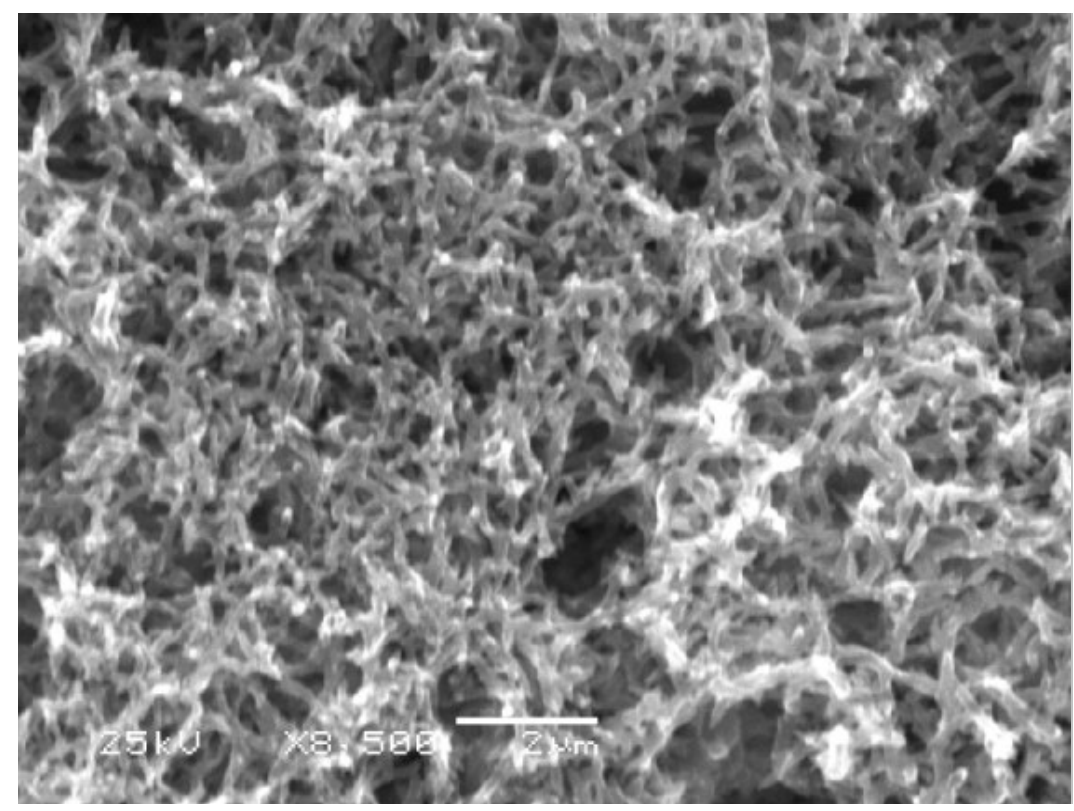

Figure 4. Scanning electron micrograph of a polyaniline-modified carbon pencil surface, obtained after 200 potentiodynamic cycles. Acceleration voltage: $25 \mathrm{kV}$, magnification: $x 8500$.

Data analysis according to Randles-Sevcik equation (Fig. 5a) indicates a departure from this model for $v>0.60 \mathrm{~V} \mathrm{~s}^{-1}$. For lower sweep rates, the RandlesSevcik equation is fulfilled, indicating that the polymer has a 2D topography. The log-log plot of the current peak and the sweep rate (Fig. 5b) yield more light to this issue. For $v \leq 0.60 \mathrm{~V} \mathrm{~s}^{-1}$, the slope equals $0.49 \pm 0.02$, corresponding to a fractal dimension of $1.98 \pm 0.04$, confirming the $2 \mathrm{D}$ topography. For $v>0.60 \mathrm{~V}$ $\mathrm{s}^{-1}$, another slope with a value of $0.88 \pm 0.04$ arises from the experimental data, corresponding to a fractal dimension of $2.76 \pm 0.08$.

\section{Discussion}

For a perfect smooth plane the expected fractal dimension is 2, and the relationship between the current peak and $v^{1 / 2}$ obeys the Randles-Sevcik equation [8]. However, for a fractal surface, that is, with $2<D_{\mathrm{f}}<3$, a modified RandlesSevcik equation that takes into account the $D_{\mathrm{f}}$ value explains the experimental results [9]. 


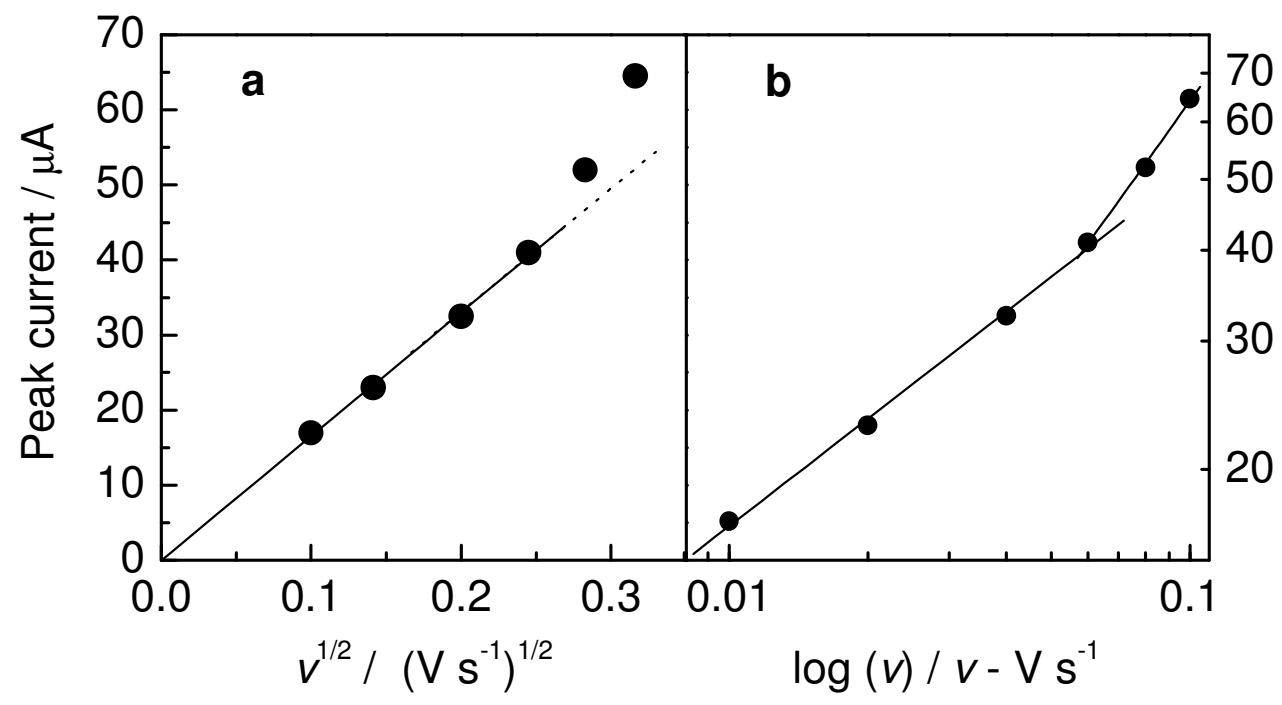

Figure 5. (a) Cathodic current vs. (sweep rate) $)^{1 / 2}$ plot for polyaniline-modified carbon pencil electrode. (b) log-log plot for data displayed in (a), for the fractal analysis according to Stromme et al. [5].

\section{Topographic characterization of naked CPE}

Although a good performance of this supporting material has been reported, it has been scarcely used for the development of electrochemical sensors [10-12].

The results obtained with both molecular probes indicate that the naked CPE behaves as a smooth 2D surface, and the same result was obtained for vitreous carbon electrodes (data not shown). This result is in accordance with the microscopic analysis of the carbon surface, as the large plane areas seen are much bigger than the molecular probe sizes. Thus, these molecules "see" a 2D plane.

\section{Topographic characterization of $\mathrm{CPE}-\mathrm{Bi}$}

Recently, Wang and co-workers have introduced the use of bismuth-coated electrodes for the stripping analysis of heavy metals [4]. This mercury-free and hence environmentally friendly electrode appears to be an attractive alternative to the mercury film electrodes.

The topographic studies indicate that Bi deposition onto CPE yields a 2D surface, indicating that such deposition does not produce a rough surface. Both the 
potentiostatic current transient and the stripping curve for deposited Bi (Fig. 3a and $3 \mathrm{~b}$, respectively) indicate that firstly a $2 \mathrm{D}$ layer of $\mathrm{Bi}$ is deposited, and then the deposit grows evenly in the third direction. It has been shown that the fast electrodeposition of metals favors surface ad-atom diffusion $[13,14]$, and under mass transfer control, rough surfaces are obtained [15]. The possibility of adatom self-diffusion towards the formation of a smooth layer is privileged on soft metals [16], and bismuth, with a melting temperature of $544 \mathrm{~K}$, can be considered as a soft metal. Consequently, ad-atom self-diffusion would be favored in such a way that 2D domains are preferentially formed over 3D protrusions, and then a smooth layer would be formed assisted also by the convective conditions of the electrodeposition process.

\section{Topographic characterization of CPE-PANI}

The fractal evaluation of surfaces depends not only on the ability of the molecular probe to approach the surface, but also on the sweep rate of the evaluation, which determines the lower cut off distance of the evaluation. The decrease in the potential sweep rate relates to a lower cut off distance, that is, the molecular probe is able to make a closer approach to the surface. In fact, the molecule size of a few Angstroms is much lesser than the measured holes of the fractal network.

In spite of the fractal topography seen by microscopic analysis, the $\mathrm{Fe}(\mathrm{CN})_{6}{ }^{3-}$ molecule is able to penetrate the network towards the underlying polymer, which is known to have a dense globular morphology approaching a 2D topography. For higher sweep rates, the molecular probe only approaches the polymer surface, sensing a fractal topography with dimension 2.76. This value confirms the fractal morphology seen by microscopic analysis, and results adequate for the adsorption of bigger molecules with surface roughness, as proteins. Thus, PANIcovered electrodes are good supporting materials for the development of biosensors, and the disposable character of the devise developed in this work makes it a suitable electrode for in-field and decentralized analyses. 


\section{Conclusions}

The use of carbon pencil as disposable electrode material provides adequate topographic characteristics upon covering with bismuth and polyaniline for stripping and biosensor-based analyses, respectively. Such characteristics can be evaluated through a simple experimental set up which includes the study of the behavior of an adequate molecular probe under semi-infinite linear diffusion conditions, and interpreting the results applying the fractal concept of topography. The new electrodes studied in this work result advantageous alternatives for the preparation of disposable electrodes in substitution of glassy carbon ones for application in-field.

\section{Acknowledgements}

CSIC (Universidad de la República, Uruguay) and PEDECIBA program (PNUD/URU) for financial support of this work.

\section{References}

1. J. Wang, Stripping Analysis, VCH Publishers, Deerfield Beach, 1985.

2. S. Petrash, T. Cregger, B. Zhao, E. Pokidysheva, M.D. Foster, W.J. Brittain, V. Sevastianov, C.F. Majkrzak, Langmuir 17 (2001) 7645.

3. B. Mandelbrot, The Fractal Geometry of Nature, W.H. Freeman \& Co., New York, 1983.

4. J. Wang, J. Lu, S.B. Hocevar, P.A.M. Farias, Anal. Chem. 72 (2000) 3218.

5. M. Stromme, G.A. Niklasson, C.G. Granqvist, Phys. Rev. B 52 (1995) 14192.

6. J. Wang, Ü.A. Kirgöz, J. Lu, Electrochem. Commun. 3 (2001) 703.

7. H. Tatsumi, K. Takagi, M. Fujita, K. Kano, T. Ikeda, Anal. Chem. 71 (1999) 1753.

8. J. Wang, Analytical Electrochemistry, John Wiley \& Sons, Inc., New York, 2000.

9. T. Pajkossy, L. Nyikos, Electrochim. Acta 34 (1989) 171. 
10. A.M. Bond, P.J. Mahon, J. Schiewe, V. Vicente-Beckett, Anal. Chim. Acta 345 (1997) 67.

11. M.H. Zahir, S. Ab Ghani, Anal. Chim. Acta 354 (1997) 351.

12. J. Wang, A.-N. Kawde, Anal. Chim. Acta 431 (2001) 219.

13. P. Herrasti, P. Ocón, R.C. Salvarezza, J.M. Vara, L. Vázquez, A.J. Arvia, Electrochim. Acta 37 (1992) 2209.

14. A.J. Arvia, R.C. Salvarezza, Electrochim. Acta 39 (1994) 1481.

15. A.J. Arvia, R.C. Salvarezza, J.M. Vara, Electrochim. Acta 37 (1992) 2155.

16. M.E. Martins, R.C. Salvarezza, A.J. Arvia, Electrochim. Acta 41 (1996) 2441. 